Archives

11 | 1993

Histoire intellectuelle // Réflexions collectives sur I'histoire sociale

\title{
Le groupe de recherches sur les exempla médiévaux
}

Jacques Berlioz et Marie-Anne Polo de Beaulieu

\section{OpenEdition}

12 Journals

Édition électronique

URL : http://journals.openedition.org/ccrh/2765

DOI : $10.4000 /$ ccrh. 2765

ISSN : $1760-7906$

Éditeur

Centre de recherches historiques - EHESS

Édition imprimée

Date de publication : 10 octobre 1993

ISSN : 0990-9141

Référence électronique

Jacques Berlioz et Marie-Anne Polo de Beaulieu, « Le groupe de recherches sur les exempla médiévaux », Les Cahiers du Centre de Recherches Historiques [En ligne], 11 | 1993, mis en ligne le 05 mars 2009, consulté le 10 décembre 2020. URL : http://journals.openedition.org/ccrh/2765; DOI : https://doi.org/10.4000/ccrh.2765

Ce document a été généré automatiquement le 10 décembre 2020.

Article L.111-1 du Code de la propriété intellectuelle. 


\title{
Le groupe de recherches sur les exempla médiévaux
}

\author{
Jacques Berlioz et Marie-Anne Polo de Beaulieu
}

1 Dans le cadre du Groupe d'anthropologie historique de l'Occident médiéval (CRH) se poursuit une enquête sur les exempla médiévaux, ces récits brefs que les prédicateurs inséraient dans leurs sermons pour convaincre leur auditoire par une leçon salutaire. L'intérêt que revêtent les exempla pour les ethnologues, les historiens et les spécialistes de littérature médiévale n'est plus aujourd'hui à souligner. Précieux pour étudier les croyances imposées aux fidèles, comme pour cerner les modèles qui devaient entrainer de leur part un changement de comportement, ils apportent aussi des informations fondamentales sur la culture des laïcs et leur vie quotidienne, et sont particulièrement utiles pour étudier les traditions « folkloriques » du Moyen Age.

\section{Aux origines de la recherche}

2 Les exempla ont bénéficié en France depuis 1968 d'une attention privilégiée dans le cadre de ce que l'on a pu appeler dans les années 1965-1968 «l'histoire des mentalités ». L'initiative de ces recherches en revient pour l'essentiel à Jacques Le Goff, abordant dès 1967 dans son séminaire de l'École pratique des hautes études ( $\mathrm{VI}^{\mathrm{e}}$ section) les problèmes des rapports entre culture savante et culture folklorique dans l'Occident médiéval. Et ce, à travers des recueils comme les Otia imperialia de Gervais de Tilbury, mort vers 1221. Plus tard, en 1974 et 1975 sont poursuivies des recherches conjointes sur l'exemplum médiéval et sur la naissance du purgatoire (XII-XIII ${ }^{\mathrm{e}}$ siècles). Une réflexion théorique sur l'exemplum est menée de pair, associant analyse de structure et de contenu, à une approche de la société médiévale à travers ses structures de l'imaginaire et l'action de l'Église, comme centre de production idéologique.

3 A partir de 1975 Jean-Claude Schmitt approfondit les rapports entre le folklore et la littérature des exempla. De 1978 à 1980, le séminaire, grâce notamment à l'intervention du sémiologue Claude Bremond, directeur d'études à l'ÉHESS, approfondit la question de la 
structure des exempla et de la logique du récit exemplaireire ${ }^{1}$. En 1978 s'inaugure un séminaire restreint, bi-mensuel, placé sous la direction de Cl. Bremond, J. Le Goff et J.-Cl. Schmitt, et consacré à la préparation d'un index des exempla médiévaux. Les travaux de cette équipe d'une dizaine de personnes complètent sur le plan technique les recherches menées par J. Le Goff et J.-Cl. Schmitt dans leur séminaire principal. C'est ce groupe qui poursuivra après 1981 les recherches consacrées spécifiquement aux exempla, J. Le Goff abordant alors la question de l'idéologie royale, autour de la personne de saint Louis.

\section{Les activités du groupe de recherches sur les exempla médiévaux}

Le caractère essentiel de ce groupe est son interdisciplinarrité. Y participent en permanence des historiens, des sémiologues, des philologues, des spécialistes des littératures italienne, espagnole, russe, arabe ou hébraïque, des sociologues ${ }^{2}$. De plus cette équipe accueille des étudiants en thèse, parmi lesquels de nombreux étrangers.

5 L'enquête menée par le Groupe se poursuit - en 1992 - dans trois directions : l'édition critique des recueils d'exempla les plus importants, tant du point de vue du fond (richesse thématique et littéraire) que de la forme (système de présentation des exempla plus ou moins élaboré, permettant l'étude d'un véritable "catéchisme exemplaire»); l'élaboration d'un système d'indexation de ces exempla; l'étude de contenu des récits exemplaires, selon des approches diverses et complémentaires. Il s'agit par là de fournir aux chercheurs une base documentaire solide, fondée sur l'érudition la plus classique ; de permettre un accès rapide et efficace à l'imposant corpus de récits exemplaires du Moyen Age ; de proposer une réflexion sur la nature et la fonction de ces récits.

\section{Éditions critiques et traductions de recueils d'exempla}

6 Dès le début de l'enquête sur les exempla médiévaux, J. Le Goff a souligné l'impérieuse nécessité d'éditions critiques. Le travail long et patient qu'exige ce type de publication fait que ce n'est qu'aujourd'hui qu'en sont offerts les premiers résultats. Ainsi, l'un des principaux recueils du Moyen Age, la Scala coeli du dominicain Jean Gobi le Jeune, vient d'être publié par M.-A. Polo de Beaulieu3. L'édition de l'imposant Tractatus de diversis materiis predicabilibus composé entre 1250 et 1261 environ par le dominicain Étienne de Bourbon est en cours sous la direction de Jacques Berlioz. Le premier volume (De dono timoris), établi par J. Berlioz et Jean-Luc Eichenlaub, est sous presse (Turnhout, Brepols, collection Corpus christianorum. Continuatio medievalis). Il est à souligner que la publication de ce volume sera accompagnée de microfiches proposant les concordances verbales du texte latin. De plus, cette édition gagnera immédiatement la banque de données CLTC (Cetedoc Library of Christian Latin Texts), diffusée par les éditions Brepols sur CD-ROM ${ }^{4}$. De même, il est probable que l'Alphabetum narrationum, recueil composé au début du XIV siècle par le dominicain Arnold de Liège, et dont Colette Ribaucourt vient d'achever l'édition, sera publié par Brepols dans cette même collection ${ }^{5}$. Ces travaux s'accompagnent de l'analyse critique de la genèse et de la transmission des exempla, afin de les situer dans la longue durée. En amont : recherche des sources antiques, orientales ou autres, pour déterminer l'originalité des récits; en aval, si possible: étude de la diffusion de ces textes dans les genres didactiques (sermons, traités didactiques religieux ou profanes, traités juridiques ou médicaux...) et de leur transformation quand ils 
pénètrent des genres littéraires comme le fabliau, la nouvelle, le roman, etc. Sur ce plan, la collaboration avec le groupe de recherche "Texte et contexte des Mille et Une Nuits " (GO 833 du CNRS) s'est avérée et s'avère très fructueuse.

D'autres éditions critiques sont en cours : les Sermones vulgares ou ad status de Jacques de Vitry $(\mathrm{XIII})$, comportant de nombreux exempla, par Marie-Claire Gasnault (EHESS) et Jean Longère; la Summula exemplorum (xIII ${ }^{\mathrm{e}}$ siècle) par Letizia Pellegrini; le recueil Florence, Riccardiano 2894, ensemble de sermons "reportés »-avec de nombreux exempla-, en italien, du Xve siècle, par Rosa Maria Dessi (EHESS) ; le Violier des histoires romaines ( $\mathrm{Xv}^{\mathrm{e}}$ siècle) par C. Velay-Vallantin; les Epistolas y Evangelios por todo el ano de Fray Ambrosio Montesino (Espagne, 1512) par Maria Matesanz. L'édition du recueil cistercien d'exempla, dit de Beaupré, datant du début du XIII ${ }^{\mathrm{e}}$ siècle, est préparée par l'ensemble du Groupe sous la direction de J. Berlioz ${ }^{6}$.

Outre les éditions critiques, le Groupe s'est attaché à la préparation de volumes de traduction d'exempla, afin de mettre à la disposition d'un public souhaité le plus large possible des textes fondamentaux du corpus ${ }^{7}$. Sont en préparation les traductions du De dono timoris du dominicain Humbert de Romans (XIII ${ }^{\mathrm{e}}$ siècle) par Gérard Blangez et JeanLuc Eichenlaub, et du Ci nous dit (xIv siècle) par Gérard Blangez.

\section{Réalisation d'un système d'indexation des exempla.}

9 La deuxième tâche prioritaire est la réalisation d'instruments de recherche destinés à rendre accessibles les recueils d'exempla du Moyen Age. Le Groupe a déjà procédé au contrôle systématique de l'instrument de recherche fondamental (mais médiocrement réalisé) : l'Index exemplorum de F. C.Tubach (Helsinki, 1969). Un travail collectif (entre 1983 et 1987) a permis d'élaborer des tables critiques de cet index, par recueil d'exempla. Ces tables, obtenues par un tri informatisé, permettent de corriger, compléter et rendre plus fiables les informations contenues dans l'Index exemplorum. Cet ouvrage, précédé d'une méthode de recherche et d'une large bibliographie est paru aux éditions GARAE/Hésiode, à Carcassonne ${ }^{8}$. La refonte de l'Index exemplorum de F. C. Tubach est entreprise actuellement dans deux directions : 1 . Un thesaurus des exempla médiévaux, sous la forme d'une banque de données ; 2) le répertoire critique des exempla italiens.

\section{Le thesaurus des exempla médiévaux}

10 Le projet de banque de données sur les récits exemplaires s'inscrit dans un mouvement général de création d'instruments de recherche regroupant les textes et les informations les concernant, dont on aura prochainement un exemple avec la diffusion du CD-ROM proposé par l'Université de Limoges et comportant l'intégralité de la poésie lyrique occitane (textes, bibliographie). Elle se fonde aussi sur l'expérience concrète de membres de notre équipe ${ }^{9}$.

11 Cette banque serait composée de deux bases de données, corrélées entre elle. La première regrouperait les informations sur chaque recueil; la seconde fournirait, exemplum par exemplum, un titre, un résumé, des mots-clés alimentant un index général, les sources ou les textes parallèles, une bibliographie. Si le choix du logiciel de gestion de cette banque de données n'est pas encore déterminé, il conviendra de permettre des interrogations souples, proches du langage nature ${ }^{10}$. Cette base sera dynamique, intégrant les nouveaux recueils au fur et à mesure de leur dépouillement ou de leur édition. Est prévu le 
traitement - en liaison étroite avec le Groupe de recherches sur les images médiévales de documents iconographiques, certains recueils d'exempla, comme le Ci nous dit, étant illustrés.

\section{Le répertoire critique des exempla italiens}

Aucun instrument de recherche ne permet actuellement d'appréhender la riche littérature exemplaire du Moyen Age en Italie. Disposer d'un répertoire critique des exempla italiens médiévaux permettra de prendre la mesure de la diffusion de la matière narrative tant en latin qu'en langue vulgaire, d'étudier les procédés de persuasion par le biais de l'exemplum dans le contexte italien et d'appréhender exactement l'influence des exempla dans la littérature profane (Boccace, Sercambi, etc.). Pour établir un tel répertoire, deux opérations parallèles sont prévus. Des chercheurs italiens, engagés dans la publication d'une édition critique des principaux recueils d'exempla italiens (à paraître chez Salerno à Rome), rédigeront dès septembre 1992 des fiches signalétiques (résumé de l'exemplum, sources, bibliographie, mots clés) pour tous les exempla des recueils dont ils ont la charge ${ }^{11}$. D'autres chercheurs, français ou italiens, analyseront de la même manière des recueils qui leur sont familiers. Les fiches établies en italien seront traduites en français puis enregistrées sur support informatique. L'ensemble fera l'objet d'une publication comprenant, outre une introduction générale sur l'exemplum en Italie, l'analyse, recueil par recueil, des exempla et de leurs sources. Un index des exempla par mots clés en permettra un accès rapide. L'École française de Rome est directement associée à cette entreprise.

\section{Étude du contenu des exempla}

La grande diversité des études menées par les membres de notre groupe se double toutefois d'une unité certaine dans les méthodes d'approche, que l'on peut schématiquement rapporter à trois :

- Des études ressortissant à l'anthropologie historique se portent tant sur la parenté et les relations familiales que sur les comportements religieux (en insistant autant que possible sur les relations entre le rituel et la croyance).

- des recherches s'attachent à l'étude du vocabulaire des exempla et utilisent les méthodes de la lexicométrie et de la statistique lexicale. Ces études permettent de saisir plus finement les procédés rhétoriques et stylistiques qui doivent contribuer à faire de $\mathrm{l}^{\prime}$ exemplum un « récit efficace ».

- un secteur de travaux relève de la narratologie, qui étudie les possibilités de segmentation des exempla en unités narratives codifiables, comparables et comptabilisables. Ceci, afin de mettre en évidence des schémas narratifs récurrents communs aux divers genres du récit.

14 Les membres du Groupe, outre leurs recherches individuelles, se rassemblent régulièrement autour d'enquêtes portant sur un sujet précis, comme en 1990 et 1991 autour du personnage de saint Bernard de Clairvaux dans les exempla ${ }^{12}$, ou en 1992 autour de la question des rapports entre encyclopédies médiévales et récits exemplaires ${ }^{13}$.

Enfin, la question théorique du statut même de l'exemplum va être reconsidérée. Dans les années 1970, principalement sous l'influence du structuralisme, les études sur la notion de genre littéraire se sont multipliées. Les nouvelles éditions critiques parues ou en cours 
comme l'attention portée par les historiens au public visé, à la réception effective des oeuvres, aux implications sociales et culturelles de l'écrit (comme à ses relations avec la tradition orale) font que la notion de genre peut faire de nouveau l'objet d'interrogations.

Pour ce faire, nous aborderons tout particulièrement les récits dont le but est a priori didactique, et qui relèvent de la littérature exemplaireire, des fables, des dits, etc. ${ }^{14}$. Pour ce qui est de l'exemplum, la question de ses définitions sera reprise, tant dans les oeuvres présentant un classement issu de la rhétorique classique (dictionnaires médiévaux; traités de rhétorique et de grammaire; arts de la prédication), que dans les ouvrages intégrant des récits exemplaireires (recueils d'exempla, sermons, ouvrages juridiques, etc.). L'essentiel sera de confronter les modèles clos et savants aux catégories exprimées par la littérature exemplaireire elle-même : l'analyse des cadres du récit comme celle des notations marginales des utilisateurs de manuscrits seront ainsi privilégiées. Il conviendra de poser également des questions jusque là éludées : ainsi, peut-on parler d' exemplum biblique au Moyen Age? quels sont les rapports entre l'exemplum rhétorique (issu de l'Antiquité) et exemplum homilétique? Comment se présentent les relations entre récits latins et exempla en langue vulgaire?

Le Groupe de recherches sur les exempla médiévaux se trouve donc, en 1993, pleinement engagé dans l'édition critique des grands recueils d'exempla du Moyen Age. L'emploi - maintenant systématique - de l'informatique, l'arrivée d'instruments de recherche comme les concordances informatisées - vont permettre d'accélérer les publications. Tout en intensifiant l'approche théorique du récit exemplaireire, le Groupe est à l'orée d'une grande entreprise : la réalisation du Thesaurus des exempla médiévaux. En un mot, de nouvelles perspectives s'ouvrent au Groupe de Recherches sur les exempla médiévaux. Un signe en est le colloque organisé ern septembre 1994 par le CRH et l'ENS de FontenaySaint-Cloud et qui portera précisément sur le thème : « Les exempla médiévaux : nouvelles perspectives. » Ce colloque sera tout d'abord l'occasion de faire le point sur les travaux menés dans le monde sur les exempla et de débattre sur la définition et la fonction des récits exemplaires. Il s'agira aussi de confronter les premiers résultats de la banque de données infirmatisée des exempla à des expériences voisines et comparables. Enfin, ce colloque aura l'ambition de proposer un programme international d'édition de textes et d'indexation.

\section{NOTES}

1. Le fruit de ce travail collectif est la parution en 1982, dans la "Typologie des sources du Moyen Age occidental » (Turnhout: Brepols) du fascicule L'« Exemplum», par Cl. Bremond, J. Le Goff et J.-Cl. Schmitt.

2. Placé sous la direction de Jean-Claude Schmitt et Claude Bremond, ses principaux animateurs en sont Jacques Berlioz (CNRS), Claude Cazalé-Bérard (Paris X-Nanterre), Bernard Darbord (Paris X-Nanterre) et Marie-Anne Polo de Beaulieu (CNRS), assistés de Colette Ribaucourt (EHESS).

3. La Scala coeli de Jean Gobi, Paris : CNRS, 1991, 768 p. (Sources d'histoire médiévale, publiées par l'Institut de recherches et d'histoire des textes). Ce recueil est important par sa taille (environ 1000 exempla), sa richesse narrative et sa grande diffusion manuscrite puis imprimée. 
4. La consultation de cette base peut par exemple se faire au Centre de recherches historiques à Paris ou au CIHAM à l'Université de Lyon II.

5. Ce recueil comporte environ 800 exempla insérés dans un système de classement alphabétique rendu très complexe par un système de renvois multiples entre les rubriques.

6. Ce recueil (Paris, BN, lat. 15912), particulièrement important pour l'étude de la diffusion des exempla monastiques (dont nombre, de tradition orale, sont inédits) dans les sermons destinés au public le plus large, sera publié aux éditions Brepols (Corpus christianorum. Continuatio medievalis). Les chercheurs associés à cette entreprise sont : J. Berlioz, A. Boureau, G. Blangez, Cl. Bremond, H. Cassaigne, B. Darbord, R. Dessi, J.-L. Eichenlaub, M. C. Gasnault, A. Launay, M.-A. Polo de Beaulieu, C. Ribaucourt, C. Velay-Vallantin.

7. Prêcher d'exemples. Récits de prédicateurs du Moyen Age présentés par J.-Cl. Schmitt, Paris : Stock/ Moyen Age, 1985 ; Formes médiévales du conte merveilleux, dir. J. Berlioz, Cl. Bremond, C. VelayVallantin, Paris : Stock/Moyen Age, 1989 ; J. Berlioz, Saints et damnés. La Bourgogne du Moyen Age dans les récits d'Étienne de Bourbon, inquisiteur (1190-1261), Dijon : Éditions du Bien Public, 1989 ; Albert Lecoy de la Marche, Le rire du prédicateur. Récits facétieux du Moyen Age. Présentation, notes et annexes par J. Berlioz, Turnhout : Brepols, 1992.

8. J. Berlioz et M.-A. Polo de Beaulieu, dir., Les exempla médiévaux. Introduction à la recherche suivie des Tables critiques de l'Index exemplorum de F. C. Tubach, Carcassonne : GARAE-Hésiode, 1992. Cet ouvrage est paru avec le concours du Centre de recherches historiques (EHESS-CNRS), de la Société de l'École des chartes (Prix Lenoir) et du Conseil général de l'Aude. Le travail informatique a été réalisé au LISH de l'EHESS.

9. Ainsi, la base de données Repertorium exemplorum de Gérard Blangez s'augmente régulièrement et compte actuellement près de 6000 fiches. Elle est pour l'instant à usage interne (voir G. Blangez, «Un répertoire des exempla », dans Mélanges Cardinal Louis-Albert Vachon, Québec: Université Laval, 1989, p. 33-42).

10. Une première maquette est testée actuellement sur le logiciel SPIRIT (Société SYSTEX) en liaison avec les Universités de Paris X-Nanterre et de Limoges.

11. Cette partie du travail est coordonnée par Claude Cazalé-Bérard.

12. J. Berlioz, Marie-Anne Polo de Beaulieu et Colette Ribaucourt, «Saint Bernard dans les exempla médiévaux ", dans Vies et légendes de saint Bernard. Création, diffusion, réception, Rencontres de Dijon, 7-8 juin 1991, Cîteaux, 1993.

13. Le résultat de ces travaux a été présenté en octobre 1992 lors d'un colloque sur l'encyclopédisme médiéval organisé à San Gimignano (Italie).

14. J. Berlioz a été chargé pour l'année 1992-1993 à l'EHESS d'une charge de conférences (« Nommer et classer les récits au Moyen Age »); ces thèmes ont été directement abordés. 